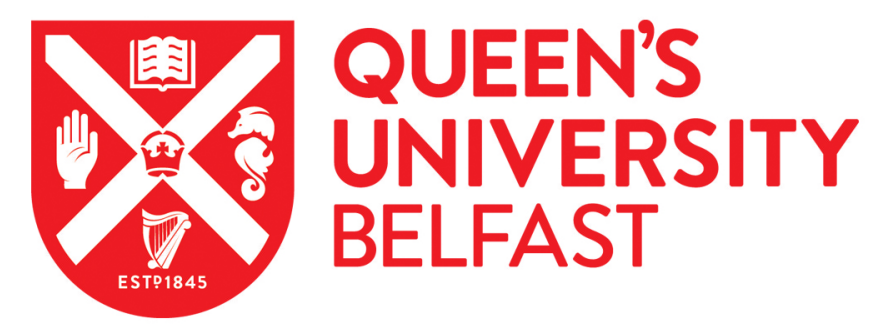

\title{
Physical Models of Wind Instruments : A Generalized Excitation Coupled with a Modular Tube Simulation Platform
}

\author{
de Bruyn, G., \& Van Walstijn, M. (1995). Physical Models of Wind Instruments : A Generalized Excitation \\ Coupled with a Modular Tube Simulation Platform. Journal of New Music Research, 24(2), 148-163. \\ https://doi.org/10.1080/09298219508570680
}

Published in:

Journal of New Music Research

Document Version:

Peer reviewed version

Queen's University Belfast - Research Portal:

Link to publication record in Queen's University Belfast Research Portal

\footnotetext{
General rights

Copyright for the publications made accessible via the Queen's University Belfast Research Portal is retained by the author(s) and / or other copyright owners and it is a condition of accessing these publications that users recognise and abide by the legal requirements associated with these rights.

Take down policy

The Research Portal is Queen's institutional repository that provides access to Queen's research output. Every effort has been made to ensure that content in the Research Portal does not infringe any person's rights, or applicable UK laws. If you discover content in the Research Portal that you believe breaches copyright or violates any law, please contact openaccess@qub.ac.uk.
} 
Gijs de Bruin

Maarten van Walstijn ${ }^{\dagger}$

Institute of Sonology

Royal Conservatory

Juliana van Stolberglaan 1

2595 CA Den Haag

$t_{\text {current address: }}$

Faculty of Music/Edinburgh Parallel

Computer Centre

University of Edinburgh

maarten@koncon.nl

gijs@koncon.nl

\section{Physical Models of Wind Instruments}

\author{
A Generalized Excitation \\ Coupled with a Modular \\ Tube Simulation Platform
}

\begin{abstract}
To develop real-time simulations of wind instruments, digital waveguides filters can be used as an efficient representation of the air column. Many aerophones are shaped as horns, which can be approximated using conical sections. Therefore the derivation of conical waveguide filters is of special interest. When these filters are used in combination with a generalized reed excitation, several classes of wind instruments can be simulated.

In this paper we present the methods for transforming a continuous description of conical tube segments to a discrete filter representation. The coupling of the reed model with the conical waveguide and a simplified model of the termination at the open end are described in the same way. It turns out that the complete lossless conical waveguide requires only one type of filter.

Furthermore, we developed a digital reed excitation model, which is purely based numerical integration methods, i.e., without the use of a lookup table.
\end{abstract}

\title{
1 Introduction
}

The last decade has seen a growing interest in the use of physical models of musical instruments for sound synthesis. The increased performance of computer systems (DSP-chips) has made real-time simulation of several classes of instruments possible.

These virtual instruments are of interest for several reasons. They are a new way of representing sound; not as a waveform or its spectrum (FFT), but as the mechanism producing it. In principle, they are expected to produce sound with an acoustic high quality (often lacking in other synthesis methods) and to respond to the gestures of a performer in a natural way, allowing for a more intuitive control of the sound. Additionally, the models can be used to study the effects of modifications of historical or contemporary instruments in a simulation; or to test acoustic theories in general, and last but not least, as a method of music synthesis. Furthermore, there is a strong relation of a "natural" sound, produced by an oscillating system liable to physical laws, with the perceptual image of such a sound. Therefore, sounds produced by physical models could be of interest in the field of sound recognition.

Our research is aimed at the development of methods for sound synthesis using these physical models, with specialisation to wind instruments. We have set us some criteria for our models, so they may be used as a basis for the development of a complete sound synthesis environment.

Firstly, the software should run in real-time, so it may be used in a performance. Secondly, the environment should be as general as possible, allowing the design of a wide variety of wind 
instruments. One way of obtaining a certain degree of generality is making the environment modular, defining a set of basic elements which can be added together to build a complete instrument. Within these two constraints, the simulation should be as faithful to acoustical laws as possible.

A general scheme for physical models is the division of a musical instrument into a linear and a non-linear part, which usually can be associated with the resonating part of the instrument and the excitation mechanism, respectively. The resonating part of an aerophone is an air column, contained in a tube system. The excitation is done by blowing (more) air in this system, by mouth or mechanically.

With the earlier mentioned criteria in mind the elements for simulating the excitation and resonator of reed-driven wind instruments were developed. This paper presents these modules, together with a method of coupling them.

\section{Excitation Mechanisms of Reed-driven Instruments}

\subsection{How a reed/tube system vibrates}

The sound production of reed-driven instruments is based on the interaction between the air column and the reed. The air column (tube) of wind instruments is usually characterized by its linear response. This response can be calculated very exactly by specifying the geometry of the air column, including the effect of toneholes, radiation and wall losses. In testing the interaction with the reed, a much more simplified model of the tube is sufficient, namely a lossless digital waveguide simulation of a cylindrical tube. The reed itself can be seen as a clamped bar (see fig. 2.1.)

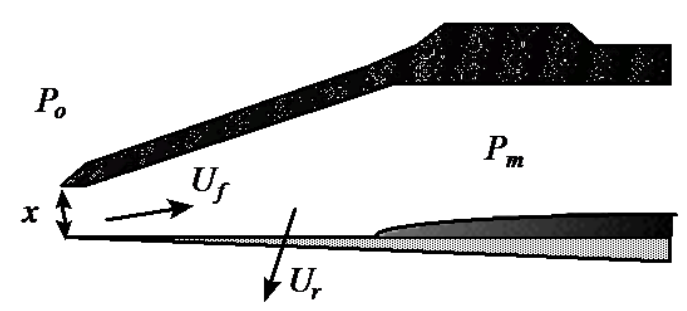

fig. 2.1 sectional model of the mouthpiece. The reed is represented by the lightgray object and is clamped to the mouthpiece. Only the movement $(x)$ of the reed tip is approximated in our model.

Typical sustained oscillations of aerophones grow because of the regenerative feedback mechanism that is inherent to any reed/tube coupling. The mouth pressure $\left(P_{o}\right)$ forced by the player causes an airflow $\left(U_{f}\right)$ through the reed aperture $(x)$. In the case of cane reed, this mouth pressure tends to close the reed, so the aperture decreases while a pressure wave $\left(p_{i}\right)$ is "released" into the tube. At the open end of the tube, a part of the wave is reflected and travels back to the mouthpiece $\left(p_{r}\right)$, where it pushes the reed back open again. Then the aperture allows a new pressure wave into the tube and the process repeats itself. Though this is a simplified version of the process, it describes the basic mechanism (see fig 2.2). The character of this interaction process is mainly determined by the physical relation between total airflow $\left(U_{b}\right)$ through the mouthpiece bore and the reed motion $(x)$.

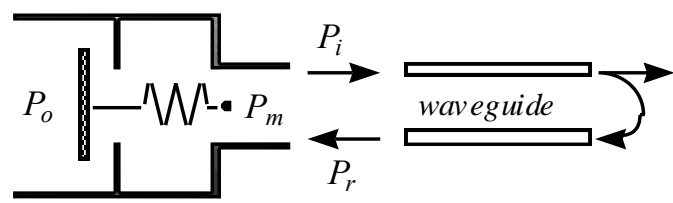

fig. 2.2 simulation model 
The motion of the reed without the influence of the player and the response of the tube is dominated by the motion of the first mode of vibration of the reed, and can therefore be modelled as a simple harmonic oscillator with a resonance frequency equal to the frequency of the first reed mode. In the model the displacement of the reed is zero when the reed is closed and is positive when opened. The flow or volume velocity is taken positive in the direction of the tube end. The reed aperture is not allowed to become negative. This corresponds to the beating of the reed when the instrument is played at higher mouth pressures. The tube response is simulated with a travelling wave or waveguide model.

\subsection{The Interaction Model.}

The equations that describe the oscillations in a reed/tube system depend on the following constants and variables that relate to physical values and quantities.

$U_{b} \quad$ Total volume flow through the bore

$U_{f} \quad$ Normal Volume flow

$U_{r} \quad$ Additional volume flow

$P_{o} \quad$ Mouth pressure

$P_{m} \quad$ Mouthpiece pressure

dP Pressure difference

$p_{r} \quad$ Reflected pressure wave from the tube

$p_{i} \quad$ Ingoing pressure wave into the tube

$x$ position of the reed (= reed aperture)

$H$ aperture of the reed in equilibrium

$Z \quad$ Characteristic impedance of the tube
$S_{t} \quad$ Cross-section of the tube

$S_{r} \quad$ Effective surface of the reed

$g_{r} \quad$ Halfpower bandwidth of the reed frequency

$w_{r}$ Reed resonance frequency

$\mu_{r} \quad$ Effective mass per unit area of the reed

$I_{r} \quad$ Inertance of the air above the reed

$c \quad$ Velocity of sound in air

$\rho \quad$ Air Density

$B$ airflow constant

The interaction between the reed motion and the tube response depends on the reed aperture and the pressure difference $(d P)$ across the reed, i.e. the difference between mouth and mouthpiece-pressure. Backus (1963) measured this flow through the reed aperture as a function of pressure difference for different rest openings and found the following expression:

$$
U_{f}=B \cdot \operatorname{sign}(d P) \cdot|d P|^{\frac{2}{3}} x^{\frac{4}{3}}
$$

The character of this relation is nonlinear due to the exponentials in the equation. The measurements were done with static reed, this means that the effect of the air inertance is neglected. If the formula is rewritten including the air inertance one obtains:

$$
d P=D \cdot \operatorname{sign}\left(U_{f}\right) \cdot\left|U_{f}\right|^{q} / x^{2}+I_{r} \cdot \dot{U}_{f}
$$

where $D=B^{-1.5}$ and $q=1.5$ for single reed and $q=2$ for double and lip reed (Keefe 1992). In this equation there is no third root like in equation (2.1) and it is therefore easier to implement on a DSP. Also the inclusion of air inertance makes it a more realistic model.

An additional flow is caused by the motion of the reed; it sweeps an extra amount of air into the tube depending on its velocity and the reed surface:

$$
U_{r}=S_{r} \cdot \dot{x}
$$

The total flow $\left(U_{b}\right)$ through the bore of the mouthpiece can now be expressed as: 
$U_{b}=U_{f}-U_{r}$

To calculate eqs. (2.2) and (2.3) the values of $x, \dot{x}$ and $d P$ have to be known. The pressure difference can be calculated simply as:

$$
d P=P_{o}-P_{m}
$$

The actual pressure in the mouthpiece is equal to the sum of the ingoing and outgoing pressure waves:

$$
P_{m}=p_{r}+p_{i}
$$

The in- and outgoing waves are related to the normalized flow in the mouthpiece:

$$
Z \cdot U_{b}=p_{i}-p_{r}
$$

with $Z$ being the characteristic impedance of the tube:

$$
Z=(\rho \cdot c) / S_{t}
$$

Now we can calculate the displacement of the reed which is depending on $d P$. Several models exist in literature, varying from simple harmonic oscillators to more complicated models of clamped bars (Stewart and Strong, 1980). Adrien (1988) used a harmonic oscillator with variable mass and stiffness coefficients, including the effect of variable reed mass and damping due to lip contact. This way an embouchure model of the reed can be developed.

According to Thompson (1979) the nonlinearity of the reed dynamics is much smaller than the nonlinearity in the flow control of the reed, and can therefore be thought of as a perturbation in the linear model. This is confirmed by Keefe (1992). and Benade (1988). Backus (1963) already found that the reed acts as a harmonic oscillator driven below its resonance frequency, although the frequency has significant influence on the tone production of wind instruments. We choose the harmonic oscillator model used by Keefe, Thompson and Benade:

$$
\ddot{x}+g_{r} \dot{x}+w_{r}(x-H)=\frac{\sigma}{\mu_{r}} d P
$$

Where $g_{r}$ is related to the damping of the reed and $\mu_{r}$ to the reed mass. The sign $\sigma=-1$ is valid for cane reed and $\sigma=1$ for lip reed, because lip reeds tend to enlarge the reed opening when the mouth pressure is increased (Keefe 1992).

The ten equations together describe the entire system (except for the tube response). To obtain all the variables at each time-step (sample period) in the discrete domain, the differential equations (2.2), (2.3) and (2.10) should be solved numerically. All the other equations can simply be integrated into the dynamical system of those three differential equations. This set is mathematically equivalent to the dynamical system described by Keefe (1992), but the elements are kept more separate. This is done to make the model easier to implement and understand.

\subsection{Solving the System in Discrete Time}

Several numerical integration methods were tried to solve the reed/tube interaction system. Due to the nonlinearities introduced by the flow characteristics and the beating of the reed the stability of the numerical integration method is essential. In the mean time the convergence of the method can not be neglected, since a physical model should have a quick response to the player's actions, similar to 
playing a real instrument. Applying pure implicit integration methods (Keefe 1992) fulfils these needs, but this also enforces enormous calculation costs, thereby prohibiting a real-time implementation. On the other hand, even with fourth-order Runge-Kutta explicit methods the desired stability could not be ensured. Therefore a special integration scheme was developed, involving both implicit and explicit methods.

The numerical integration results in a single nonlinear equation:

$$
D_{1} \cdot U_{f}+D_{2} \cdot \operatorname{sgn}\left(U_{f}\right) \cdot\left|U_{f}\right|^{q}+D_{3}
$$

where $D_{1}, D_{2}, D_{3}$ are the products of variables in the system, but can be considered as constants in this equation. From this equation $U_{f}$ is solved using the Newton-Rhapson iteration method.

\subsection{Numerical Results}

The first results were obtained by connecting a single reed model with a digital waveguide, representing a cylindrical tube, which resulted in clarinet-like oscillations. Fig. 2.3 shows a waveform of the first 1024 samples. The cylindrical shape of the tube with one open end (the side where the reed is connected can be considered as a closed end) forces the sustain of uneven harmonics. This is shown in fig. 2.4.

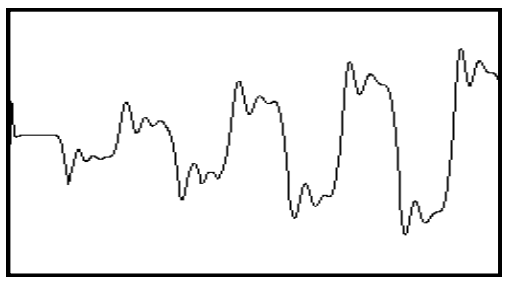

fig. 2.3 clarinet-like simulation waveform

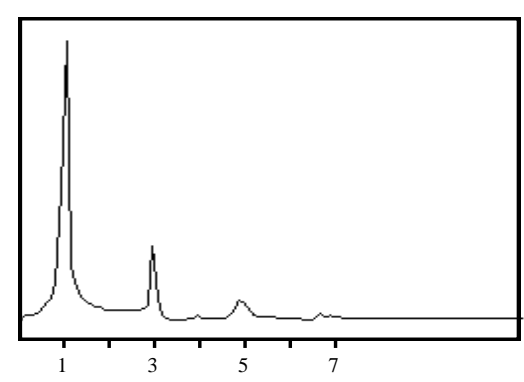

fig 2..4 spectrum of 2048 samples. On the horizontal the harmonic number is shown.

\section{Digital waveguide Modelling of Acoustical Tubes}

\subsection{Introduction}

Modelling with digital waveguide filters (hereafter refered to as waveguides) is a particularly efficient method for synthesizing the sound of musical instruments (Smith, 1993). The method is based on the solution of the one-dimensional wave equation in travelling waves. The travelling waves are represented by two parallel delay-lines (rails) propagating in opposite direction. Waveguides are closely related to classical lattice filters and transmission line descriptions of acoustical systems.

The wave propagation in cylindrical and conical tubes can be simulated with a single waveguide system. Many air columns are not perfectly cylindrical or conical, but are shaped as horns. The horn shape can be approximated with conical sections. Impedance changes occur at the cone junctions and at the open end (termination) of the air column. These impedance changes are simulated by placing filters in the delay-line system. (see fig. 3.1). 


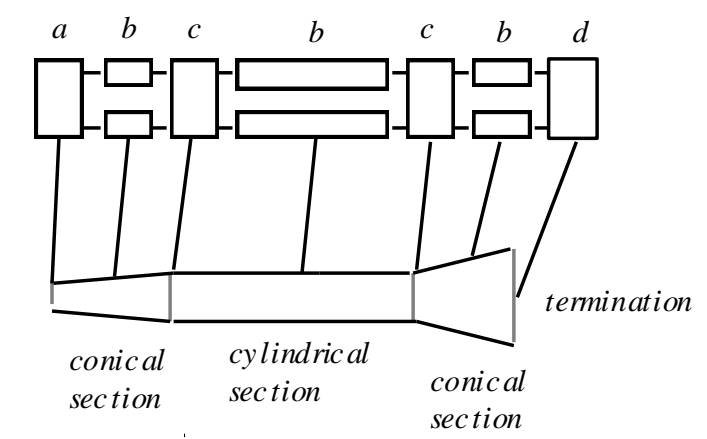

fig. 3.1 waveguide representation of an air column. The letters represent the following elements: (a) reed coupling filter. (b) waveguide. (c) scattering junction. (d) termination junction.

The waveguide junctions are called scattering junctions. For our model a special termination junction was developed, as well as a waveguide filter for the coupling of a reed excitation model with a conical tube. Thermo-viscous losses can be introduced in the system by including special filters (Agulló et al., 1993 , Amir et al., 1994). This is not yet included in our model.

\subsection{Conical Waveguides}

The one dimensional wave equation for spherical waves in a conical tube is:

$\frac{1}{r^{2}} \frac{\partial}{\partial r}\left(r^{2} \frac{\partial P}{\partial r}\right)=\frac{1}{c^{2}} \frac{\partial^{2} P}{\partial^{2}}$

Where $P$ stands for pressure as a function of time $(t)$ and place $(r)$, with $r=x+x_{0}$, the distance from the cone apex (see fig. 3.2).

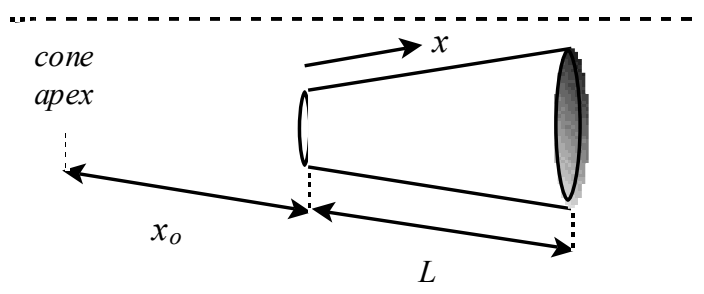

fig. 3.2 A conical air column.

Solution of the wave equation in travelling waves reveals the waveguide representation:

$P(r, t)=\frac{p^{+}}{r}+\frac{p^{-}}{r}$

with $\left\{\begin{array}{l}p^{+}=A e^{-j k r+j \omega t} \\ p^{-}=B e^{j k r+j \omega t}\end{array}\right.$

where $p^{+}, p^{-}$represent the pressure wave components in positive (upper waveguide rail) and negative (lower waveguide rail) direction. The solution in volume velocity waves in the cone yields: 
$U(r, t)=\frac{u^{+}}{r}-\frac{u^{-}}{r}$

with $\left\{\begin{array}{l}u^{+}=Z_{x}^{-1}\left(1-\frac{j}{k r}\right) A e^{-j k r+j \omega t} \\ u^{-}=-Z_{x}^{-1}\left(1+\frac{j}{k r}\right) B e^{j k r+j \omega t}\end{array}\right.$

Where wave propagation $k=\omega / c$, and with the standard characteristic impedance $Z_{x}=\rho \cdot c / S_{x}$ at point $x$ of the air column.

From these equations we can derive the local characteristic impedance. Impedance $(Z)$, or the reciprocal value of impedance, admittance $(Y)$, is a frequency dependent value that indicates the resistance to motion at a certain place in the system. In acoustical systems the definitions are:

$$
\begin{aligned}
& Z=P / U \\
& Y=U / P
\end{aligned}
$$

In cylindrical tubes the characteristic impedance is a constant value depending on the tube diameter. In conical tubes the impedance also is a function of frequency and location. Kinsler \& Frey (1950) give the admittances for upper and lower rail. They can be calculated from eqs. (3.2) , (3.4) and (3.6):

$$
\begin{aligned}
& Y^{+}=Z_{x}^{-1}(1-j / k r) \\
& Y^{-}=Z_{x}^{-1}(1+j / k r)
\end{aligned}
$$

Note that these expressions are also valid for the cylindrical case, because then $r \rightarrow \infty$.

From the characteristic impedances we can calculate the coordinate transformation from transmission line coordinates to waveguide coordinates. The transformation translates a representation in pressure $(P)$ and flow $(U)$ values to upper $\left(p^{+}\right)$and lower $\left(p^{-}\right)$travelling waves. Combining (3.3) and (3.7) gives:

$$
\begin{aligned}
& p^{+}=\frac{(1+j / k r) P+Z_{x} U}{2 r} \\
& p^{-}=\frac{(1-j / k r) P-Z_{x} U}{2 r}
\end{aligned}
$$

The transformation is quite similar to the one used in wave digital filters (Lawson and Mirzai, 1990), using the equivalents of pressure and flow in electrical circuits, voltage and current. The main difference is that within the wave digital filter transformation no frequency dependent resistance can be defined. The frequency dependency is assimilated in adaptors, which represents an element in a electrical circuit model of the acoustical system. These models are widely available in literature (Benade, 1988), (Stewart and Strong, 1980), from which wave digital filter simulations could be made. We choose to use waveguide filter representations, because they are more directly related to acoustical systems.

\subsection{Reed Coupling}

The above transformation is necessary for the coupling of the reed model with a conical waveguide, since the reed model is calculated in transmission line parameters; it gives flow as output, which has to 
be translated into waveguide coordinates. An equation can be derived that expresses the coupling between the reed and the tube involving one filter (see fig. 3.3 for signal flow):

$$
p^{+}=Z_{o} U+p^{-}-R_{c}(\omega)\left[Z_{o} U+2 p^{-}\right\rfloor
$$

where $R_{c}(\omega)$ is a first order lowpass filter:

$$
R_{c}(\omega)=\frac{\alpha_{c}}{j \omega+\alpha_{c}}
$$

with $\quad \alpha_{c}=c / x_{o}$

So the coupling filter transfer function only depends on the distance to the cone apex $\left(x_{o}\right)$. In the cylindrical case there is no filtering, because then $x_{o} \rightarrow \infty$ and $R_{c}(\omega) \rightarrow \infty$, which corresponds to equation (2.7).

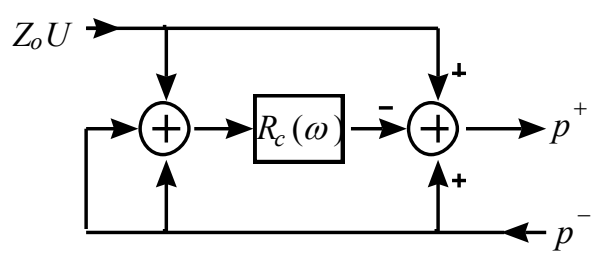

fig 3.3 reed coupling signal flow.

\subsection{Cone Junctions}

The filters structures that represent the coupling of two tube sections are called scattering junctions. An example junction of the cones $a$ and $b$ is depicted in fig. 3.4. The conical scattering equations can be derived by following Kirchoff's law, which expresses the condition of conservation of volumetric flow and pressure. The equations are expressed in reflection coefficients $\left(R^{ \pm}(\omega)\right)$ and transmission coefficients $\left(T^{ \pm}(\omega)\right)$. The reflection coefficient indicates how much of a travelling wave is reflected, whereas the transmission coefficient represents the part of the signal that is guided through the junction.

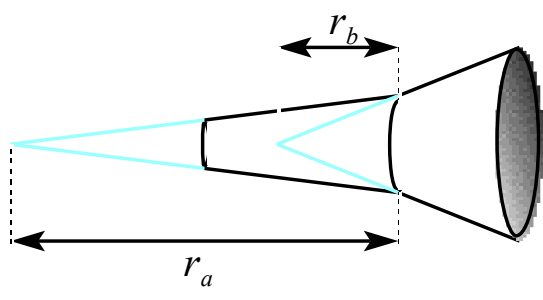

fig. 3.4 Junction of two cones

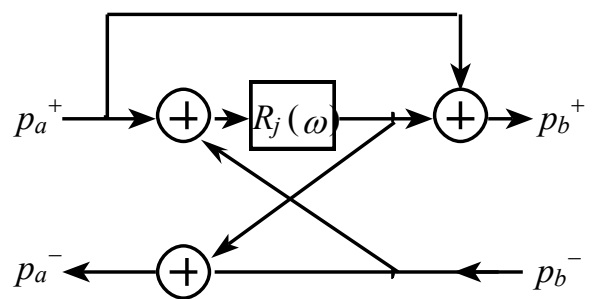

fig 3.5 conical scattering junction without taper discontinuity

Välimäki (1994a) and Agulló (1993) have derived the general conical scattering equations, which involve two filters. We are mainly interested in junctions with equivalent cone diameters, since most instrument air columns do not have taper discontinuities. Fortunately in this case there is only one filter necessary: 


$$
\begin{aligned}
& p_{a}{ }^{-}=p_{b}{ }^{-}+R_{j}(\omega)\left(p_{a}{ }^{+}+p_{b}{ }^{-}\right) \\
& {p_{b}}^{+}=p_{a}{ }^{+}+R_{j}(\omega)\left(p_{a}{ }^{+}+{p_{b}}^{-}\right)
\end{aligned}
$$

This filter is the same first order lowpass type as $R_{c}(\omega)$ :

$$
R_{j}(\omega)=\frac{-\alpha_{j}}{j \omega+\alpha_{j}}
$$

with $\alpha_{j}=\frac{c\left(r_{a}-r_{b}\right)}{2 r_{a} r_{b}}$

One (or both) of the tubes may be cylindrical, for example when $r_{a} \rightarrow \infty$, then $\alpha_{j}=c / 2 r_{b}$. Furthermore, the scattering junction is valid for both tapering $\left(x_{o}<0\right)$ and flaring $\left(x_{o}>0\right)$ cones (Välimäki 1994b).

\subsection{Termination}

The radiation impedance of the spherical wave can be used as an approximation of the real termination impedance at the open end. Comparison with the radiation formulas given by Levine and Schwinger (1948) shows that this impedance is not correct in the lower frequencies. Nevertheless it is a useful test model that represents the basic acoustic mechanism. Fletcher and Rossing (1991) give the impedance of a spherical wave with end radius $a_{l}$ :

$$
Z_{l}=Z_{e} \frac{j k a_{l}+\left(k a_{l}\right)^{2}}{\left(k a_{l}\right)^{2}+1}
$$

From this equation we can calculate the termination admittance:

$$
Y_{l}=Z_{e}^{-1}\left[1+\frac{1}{j k a_{l}}\right]
$$

This admittance is equal to the admittance of an infinite cone with an apex distance $r_{l}$ equal to the radius $a_{l}$. This means that, within our model, we can consider the impedance transition from a cone to the open air as a junction of that cone with an infinite cone (see fig 3.6). There is no reflection at the end of an infinite cone, so the radiation scattering junction only reflects $p_{a}{ }^{+}$:
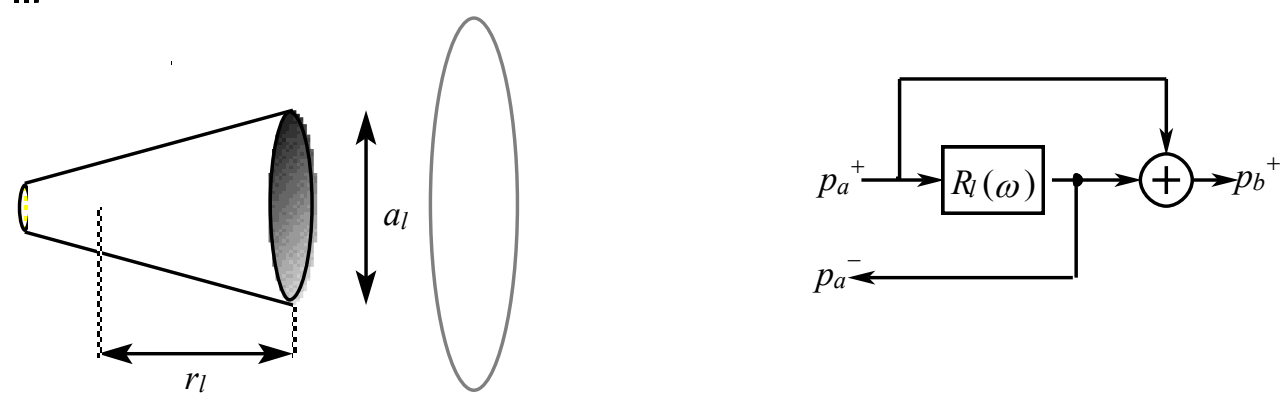

fig. 3.6 Termination model: junction of a cone

fig. 3.7 Termination scattering junction. with an infinite cone junction. 


$$
\begin{aligned}
& p_{a}{ }^{-}=R_{l}(\omega) p_{a}{ }^{+} \\
& p_{b}{ }^{+}=p_{a}{ }^{+}+R_{l}(\omega) p_{a}{ }^{+}
\end{aligned}
$$

$R_{l}(\omega)$ is the same as $R_{j}(\omega), \alpha_{l}=\frac{c\left(r_{a}-a_{l}\right)}{2 a r_{a}}$ :

$$
R_{l}(\omega)=\frac{-\alpha_{l}}{j \omega+\alpha_{l}}
$$

\section{6 Discretisation}

The complete model requires only one type of analog filter :

$$
H(s)=\frac{-\alpha}{s+\alpha}
$$

(with $s=j \omega$ )

This filter has to be mapped into the discrete domain. Since we are simulating a physical system, the transformation should map both the magnitude and the phase properties as correct as possible to the Z-domain. We made a comparison between the impulse-invariance method and the bilinear transform of this filter type. The magnitude response of the bilinear transform underestimates that of the analog filter, while the impulse-variation method causes an overestimation. Both methods have quite accurate (error of less than $3 \mathrm{~dB}$ ) magnitude responses up until $10 \mathrm{kHz}$.

The impulse-invariant method causes a linear phase component of half a sample period in the phase response. This means that the waveguide on which a filter is placed becomes half a sample longer. If more filters are used the total extra delay must be compensated within the waveguide. With the use of the bilinear transform this problem does not arise. This allows a more intuitive waveguide filter implementation.

\subsection{Fractional Delay Waveguides}

To be able to connect tubes of arbitrary length the number of delays required in the waveguide simulation is not fitting to the integer delay-steps provided by the waveguide implementation. Therefore interpolation and deinterpolation techniques must be used to obtain fractional delay values (Välimäki, 1994b). In our current model we use a first order allpass filter for this interpolation.

\subsection{Conclusions}

A real-time simulation model of reed-driven instruments is developed by coupling a reed model to a waveguide simulation of acoustical tubes. The shape of these tubes is approximated with conical sections. The effects of impedance transitions are simulated with filters. In our current model the complete waveguide can be modelled with only one type of filter.

A general digital reed model has been developed without using a lookup table as is usually done in implementations, that yet includes the effects of dynamical reed, reed inertia and reed beating. The model does not only show a good steady state behaviour and frequency domain characteristics that match with existing instruments, it also is capable of simulating authentically the fast transients, which occur at every parameter change caused by a player. It is the opinion of the authors that these transients reveal a great deal of the characteristic 'acoustic' nature of instruments.

What is excluded from this model is the complex effect of turbulent airflow, which is, for example, an 
important effect in clarinets according to Hirschberg (1994). Another simplification is the representation of the reed: its motion is reduced to the influence of the first mode. Furthermore, frequency dependent losses can be included in a future model.

The major advantage of the model lies in the generality, because of the modular structure of the waveguide representation and the flexibility of the reed simulation: within the same DSP-model single reed, double reed and lip reed can be simulated.

\section{References}

Adrien, J.M. (1988). Dynamical modelling of stringed and wind instruments, Sound synthesis by physical models. In Proceedings of International Computer Music Conference 1988 (pp. 265-276).

Amir, N., G. Rosenhouse, U. Shimony, and I. Shelef. (1994). Losses in Tubular Acoustic Systems - Theory and Experiment in the sampled Time and Frequency Domains. In Proceedings of International Computer Music Conference 1993 (pp. 384-386). San Francisco: International Computer Music Association.

Agulló, J., S. Cardona, and D.H. Keefe. (1993). Time-domain measurements of reflection functions for discontinuities in wind-instrument air columns. In Proceedings of the Stockholm Music Acoustics Conference (SMAC 93) (pp. 465-469), Stockholm : Royal Swedish Academy of Music.

Backus. (1963). Small-Vibration Theory of the Clarinet. Journal of the Acoustical Society America 35(3) (pp. 305-313).

Benade, A.H., (1988). Equivalent circuits for conical waveguides. Journal of the Acoustical Society America 83b(5) (pp. 1764-1769).

Benade, A.H., and S.N. Kouzoupolis. (1988). The clarinet spectrum: Theory and experiment. Journal of the Acoustical Society America 83(1) (pp. 292-304).

Fettweis, A. (1972). Digital Filter Structures Related to Classical Filter Networks. Selected Papers in Digital Signal Processing II. New York: IEEE Press. Reprint from Trans. Circuit Theory CT-19 (pp. 669-673).

Fletcher, N.H, and T.D. Rossing. (1991). The Physics of Musical Instruments. New York: Springer Verlag.

Gray, A.H. jr., and J.D. Markel. (1976). Linear Prediction of Speech. New York: Springer-Verlag.

Hirschberg, A., J. Gilbert, A.P.J. Wijnands, and A.M.C. Valkering. (1994). Musical aero-acoustics of the clarinet. Fifth French Conference on Acoustics Toulouse.

Keefe, D.H. (1992). Physical Modeling of Wind Instruments. Computer Music Journal, 16(4), 1992 (pp. 57-73). MIT.

Keefe, D.H. (1990). Woodwind air column models. Journal of the Acoustical Society America 88(1) (pp. 35-51).

Kinsler, L.E., and A.R. Frey. (1950). Fundamentals of Acoustics. New York: John Wiley \& sons.

Lawson, S., and A. Mirzai. (1990). Wave Digital Filters. New York: Ellis Herwood.

Levine, H., and Schwinger, J. (1948). On the radiation of sound from an unflanged pipe. Phys. Rev. 73 (pp. 383-406).

Rochesso, D., and F. Turra. (1993). A generalized excitation for real-time sound-synthesis by physical models. In Proceedings of the Stockholm Music Acoustics Conference (SMAC 93) . (pp. 584-587). Stockholm: Royal Swedish Academy of Music.

Smith, J.O. (1993). Physical Modeling using Digital Waveguides. Computer Music Journal 16(4) (pp. 75-87).

Smith, J.O. (1991). Waveguide Simulation of Non-cylindrical Acoustic Tubes. In Proceedings of International Computer Music Conference 1991. San Francisco: International Computer Music Association.

Stewart, S.E., and W.J. Strong. (1980). Functional model of a simplified clarinet. Journal of the Acoustical Society America 68(1) (pp. 109-120).

Thompson, S.C. (1979). The effect of the reed resonance on woodwind tone production. Journal of the Acoustical Society America 66(5) (pp. 1299-1307).

Välimäki, V., and M. Karjalainen. (1994a). Digital Waveguide Modeling of Wind Instrument Bores Constructed of Truncated Cones. In Proceedings of International Computer Music Conference 1994 (pp. 423-430). San Francisco: International Computer Music Association.

Välimäki, V. (1994b). Fractional Delay Waveguide Modelling of Acoustic Tubes. Report 34, Helsinki University of Technology. 